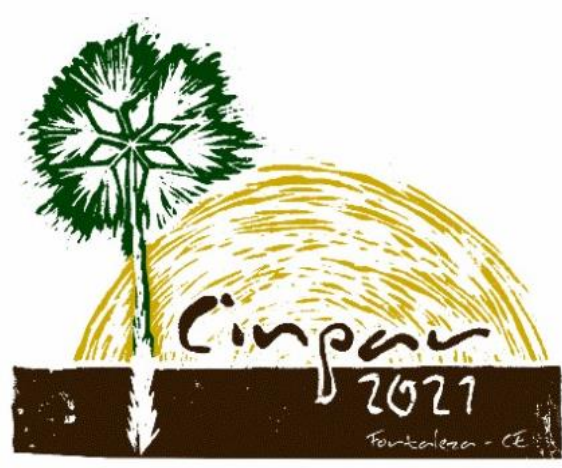

XVII Congresso Internacional sobre Patologia e

Reabilitação das Construções

XVII Congreso Internacional sobre Patología y Rehabilitación de las Construcciones

XVII International Conference on Pathology and Constructions Rehabilitation

FORTALEZA (Brasil), 3 a 5 de junho de 2021

https://doi.org/10.4322/CINPAR.2021.022

\title{
Parâmetros de gestão para reabilitação em Obras de Arte Especiais
}

\section{Management parameters for rehabilitation in bridges and viaducts}

\author{
Rodrigo Pereira ${ }^{1}$, Pedro H. Lucena ${ }^{2}$, Ana C. V. Salles ${ }^{3}$, Caroline P. Alves ${ }^{4}$, Giovana A. de Oliveira ${ }^{5}$ \\ ${ }^{1}$ PUC-MG, Belo Horizonte, Brasil, rodrigoestruturas@gmail.com \\ 2 UFMG, Belo Horizonte, Brasil, pedrohenri_que@outlook.com \\ ${ }^{3}$ UFMG, Belo Horizonte, Brasil, anasalles1304@gmail.com \\ ${ }^{4}$ UFMG, Belo Horizonte, Brasil, caroline.pedrosaalves@gmail.com \\ ${ }^{5}$ UFMG, Belo Horizonte, Brasil, abreu_giovana@hotmail.com
}

\begin{abstract}
Resumo: A gestão dos elementos que compõem a rede viária é de extrema importância para a manutenção do setor de transportes do país. Ademais, não há por parte dos órgãos públicos, principalmente a níveis estadual e municipal, uma política rígida que estabeleça diretrizes para a realização de vistorias periódicas de danos e manifestações patológicas existentes nessas estruturas, dificultando o diagnóstico e o estabelecimento de ações de recuperação e reabilitação. Dessa forma, o presente trabalho tem como objetivo instituir parâmetros qualitativos e quantitativos, baseados em condições de estabilidade e preservação, necessidade de reabilitação, grau de susceptibilidade a riscos e grau de importância junto ao sistema viário de Obras de Arte especiais (OAEs), que servirão de arcabouço teórico para a orientação de ações políticas de conservação e manutenção preventiva por parte de entidades governamentais administradoras da malha rodoviária. Para isso, foi realizado um estudo de caso avaliando as condições de 4 pontes, a partir do emprego das técnicas de avaliação propostas. Finalmente, conclui-se sobre a importância da combinação de diferentes aspectos a fim de possibilitar uma avaliação eficiente a respeito das condições das OAEs.
\end{abstract}

Palavras-chave: Transportes; Obras de Arte Especiais; Infraestrutura; Gestão.

Abstract: Managing the elements that make up the road network is extremely important for maintaining the country's transport sector. Furthermore, there is no rigid policy on the part of public bodies, especially at the state and municipal levels, which establishes guidelines for conducting periodic inspections of damage and pathological manifestations existing in these structures, hindering the diagnosis and establishment of recovery actions. The present work aims to establish qualitative and quantitative parameters, based on conditions of establishment and preservation, need for rehabilitation, degree of susceptibility to risks and degree of importance within the road system of bridges and viaducts adapted to the Brazilian reality, which will serve as a theoretical framework for the orientation of political actions of conservation and preventive maintenance by the governmental bodies that manage the road network. There will be a case study of four bridges in which it will be used as methods developed throughout the work. Finally, it is possible to conclude about the importance of combining different aspects to provide an efficient evaluation of the bridges and viaducts conditions.

Keywords: Transport; Bridges and viaducts; Infrastructure, management.

\section{Introdução}


As Obras de Arte Especiais (OAEs) são componentes essenciais da infraestrutura viária de um país. As pontes são fundamentais para a ligação entre diferentes localidades, permitindo vencer vãos de cursos d'água. Já os viadutos facilitam o tráfego entre vias em diferentes níveis e possibilitam interseções.

Como qualquer obra de engenharia, as OAEs possuem uma vida útil que varia conforme os impactos físicos e bióticos que sofrem ao longo do tempo. Esses impactos resultam em manifestações patológicas nos elementos das OAEs, que, com o tempo, podem levar ao colapso da estrutura, sendo um risco para os usuários (VITÓRIO, 2006).

A malha rodoviária brasileira começou a ser implantada efetivamente a partir da década de 40 , tendo sua maior expansão entre os anos de 1960 e 1980. Com o passar dos anos, várias obras de duplicação foram realizadas nas rodovias e fizeram com que intervenções para alargamento e reforço fossem necessárias nas OAEs existentes. Assim, existe uma grande variedade de características construtivas das OAEs no país, que nem sempre estão adequadas ao volume de tráfego e capacidade de carga atual. Dessa forma, observa-se que grande parte das manifestações patológicas identificadas nas OAEs é devido ao longo tempo de existência da estrutura e a ausência de serviços de reabilitação e recuperação dos elementos (CARVALHO, 2018; VITÓRIO, 2013).

Vitório (2007), ao analisar diferentes casos de colapsos de pontes que ocorreram no Brasil entre 1990 e 2005, concluiu que a ausência de procedimentos de acompanhamento das obras e a não realização de manutenções periódicas são alguns dos fatores que influenciaram nos acidentes.

Nos Estados Unidos, o colapso de uma ponte em 1967 levou à criação de um órgão nacional de inspeção de pontes. Desde então, foram desenvolvidos sistemas de gerenciamento de OAEs com o objetivo de monitorar as condições de conservação e estabilidade das obras do país. Outros países também adotam seus sistemas de gerenciamento, permitindo o acompanhamento das OAEs e a gestão da necessidade de manutenções preventivas e corretivas (MENDES, 2009).

No Brasil, a NBR 9452:2019 e o Manual de Inspeção de Pontes Rodoviárias são os documentos que guiam as inspeções realizadas nas OAEs brasileiras. O Departamento Nacional de Infraestrutura de Transportes (DNIT) administra o Sistema de Gerenciamento de Obras de Artes Especiais (SGO), que depois de um período sem atualizações, voltou à atividade nos últimos anos, recebendo novas vistorias periódicas das OAEs localizadas em rodovias federais (MOSCOSO, 2017).

$\mathrm{O}$ presente artigo tem como objetivo estabelecer parâmetros que orientem quanto à necessidade de reabilitação de OAEs brasileiras. Para isso é apresentado um estudo de caso de 4 obras inspecionadas no ano de 2020, avaliando as notas atribuídas nas inspeções, características construtivas e deficiências funcionais identificadas, como forma de definir aspectos que possam ser considerados prioritários no âmbito da gestão de reabilitação de OAEs no país.

\section{Estudos Internacionais}

A preocupação com a integridade do sistema de transportes terrestres é comum aos governos em todo o mundo. Dessa forma, diversos países adotam sistemas de gerenciamento de OAEs, que se adaptam conforme a realidade local, e auxiliam a tomada de decisão quanto à necessidade de recuperação das estruturas ao longo do tempo.

Nos EUA, são utilizados os softwares Pontis e Bridgit que permitem acompanhar as condições de preservação das OAEs ao longo do tempo e visam aumentar a vida útil da estrutura, garantindo segurança e custos reduzidos de manutenção (MOSCOSO, 2017).

Na Alemanha, o BASt (Federal Highway Research Institute) realiza inspeções nas OAEs para identificação de danos periodicamente e, a partir disso, aplica as medidas de recuperação necessárias. $O$ instituto administra um sistema que avalia, a partir do banco de dados, as condições, custos e alternativas a serem aplicadas nas estruturas (MENDES, 2009).

$\mathrm{Na}$ França, o Cerema (Centre d'études et d'expertise sur les risques, l'environnement, la mobilité et I'aménagement) disponibiliza guias para a gestão das OAEs no país. A partir do documento Surveillance et 
entretien courant des ouvrages d'art routiers, são definidos os diferentes tipos de inspeção e métodos de avaliação das OAEs. Por exemplo, o método IQOA (Image Qualité des Ouvrages d'Art) estabelece critérios para atribuir classes para as obras, que indicam o estado de conservação e estabilidade, bem como a necessidade de reparação da estrutura (CEREMA, 2018).

O Reino Unido possui programas de avaliação de pontes desde 1960. Desde então, foram desenvolvidos sistemas para gerenciar as OAEs do país ao longo do tempo. Atualmente, a Highways Agency (HA) é o órgão responsável pelo gerenciamento das pontes britânicas (MENDES, 2009). O Structures Management Information System (SMIS) é o sistema onde foram registradas as informações das inspeções realizadas nas obras ao longo dos últimos 20 anos. No entanto, em 2019 a HA migrou as informações e passou a usar um sistema mais tecnológico, o AssetWise, para auxiliar as inspeções das obras e orientar quanto às medidas de manutenção e reparação (ASHWELL, 2019; BENTLEY, 2020).

Recentemente, na Europa foi desenvolvido um estudo pelo COST (European Cooperation in Science \& Technology), o COST ACTION TU1406, que buscou estabelecer e padronizar critérios de qualidade para as pontes rodoviárias europeias. A partir desse estudo, realizado de forma colaborativa entre mais de 38 países, foram determinados indicadores de qualidade das OAEs, que consideram diversos fatores como a análise do ciclo de vida, mudanças climáticas, desempenho sísmico, fatores sociais e econômicos, a facilidade de manutenção, confiabilidade e resiliência das estruturas.

\section{Inspeção de Pontes e Viadutos}

A base de todo sistema de gerenciamento de OAEs requer um banco de dados que permita extrair as informações necessárias para as tomadas de decisão. Para isso, são necessárias as inspeções das obras, que têm como objetivo registrar as características das OAEs e avaliar manifestações patológicas visíveis.

Como informado no tópico anterior, cada país adota determinados procedimentos de inspeção e registro dos dados. No Brasil, a NBR 9452:2019 - Inspeção de pontes, viadutos e passarelas de concreto (ABNT, 2019) define os diferentes tipos de inspeção em OAEs, sendo eles:

- Inspeção cadastral: É a primeira inspeção realizada na obra e tem como objetivo inserir as informações no sistema de gerenciamento. O ideal é que seja realizada imediatamente após a conclusão da obra. Na inspeção cadastral são relevantes as informações da geometria da OAE e o conhecimento dos elementos que a compõem.

- Inspeção rotineira: É uma inspeção periódica, realizada em determinado intervalo de tempo, que tem como objetivo analisar visualmente os elementos e condições da estrutura. Durante uma inspeção rotineira é avaliado o surgimento ou evolução de manifestações patológicas, sendo registrados em fotos e anotações.

- Inspeção especial: De acordo com a norma, a inspeção especial é realizada em OAEs que foram avaliadas com nota crítica e necessidade imediata de intervenção ou nos casos que a obra irá passar por procedimentos de adequação do sistema estrutural, como alargamentos, reforços ou prolongamentos.

- Inspeção extraordinária: É realizada a partir do surgimento de demandas não programadas, como impacto de veículos, ocorrência de fenômenos naturais como inundações ou então a partir da necessidade de verificar determinado elemento com maior atenção.

Além dos tipos de inspeção, a norma propõe uma classificação de condição da estrutura de acordo com seus parâmetros estruturais, funcionais e de durabilidade, sugerindo a atribuição de notas que variam de 1 a 5 , sendo nota 1 atribuída para condições críticas e 5 para condições excelentes.

O Manual de Inspeção de Pontes Rodoviárias é um documento disponibilizado pelo DNIT que tem como objetivo padronizar os procedimentos de inspeções em OAEs no Brasil. O manual atribui responsabilidades e deveres para o inspetor de pontes e descreve de forma detalhada os parâmetros que devem ser observados durante as inspeções, como a identificação dos elementos e do sistema construtivo.

Além dos tipos de inspeção apresentados na NBR 9452:2019 (ABNT, 2019), o manual de inspeção apresenta um quinto tipo, a inspeção intermediária, que tem como objetivo o monitoramento em menor prazo de 
anomalias identificadas nas inspeções anteriores. Ademais, o documento auxilia a identificação de manifestações patológicas e descreve aquelas mais comuns observadas nos diferentes elementos e materiais.

Um outro ponto importante abordado no manual é a possibilidade de identificar o ano de construção das OAEs com base nas características da sua seção transversal. Como muitas vezes faltam informações sobre os projetos e execução das obras, essa é a melhor forma de conhecer o período de construção da estrutura e, consequentemente, seu tempo em atividade. Além disso, também é possível extrair a partir das características construtivas da obra a capacidade de cargas móveis para a qual foi projetada, sendo esses dados importantes para agregar ao cadastro das OAEs.

As inspeções realizadas nas rodovias federais, sob jurisdição do DNIT, são registradas no Sistema de Gerenciamento de Obras de Artes Especiais (SGO), onde são atribuídas as notas de estabilidade e conservação para as estruturas inspecionadas, conforme a NBR 9452:2019 (ABNT, 2019).

\section{Gestão de OAEs}

É de interesse dos governantes e da população que acidentes com OAEs sejam evitados, sendo a estabilidade e integridade das estruturas alguns dos fatores determinantes para a garantia de segurança da comunidade e dos usuários. Além disso, também deve ser levada em consideração a redução de custos quando uma OAE tem sua vida útil prolongada e não necessita de substituições.

De acordo com Possan e Demoliner (2013), a vida útil de uma estrutura está relacionada à realização de manutenções periódicas, como ilustrado no gráfico da Figura 1. Por isso, a existência de um sistema de gestão de OAEs pode ser determinante para o aumento da vida útil das estruturas e da garantia de estabilidade, uma vez que permite gerenciar a necessidade de manutenção.

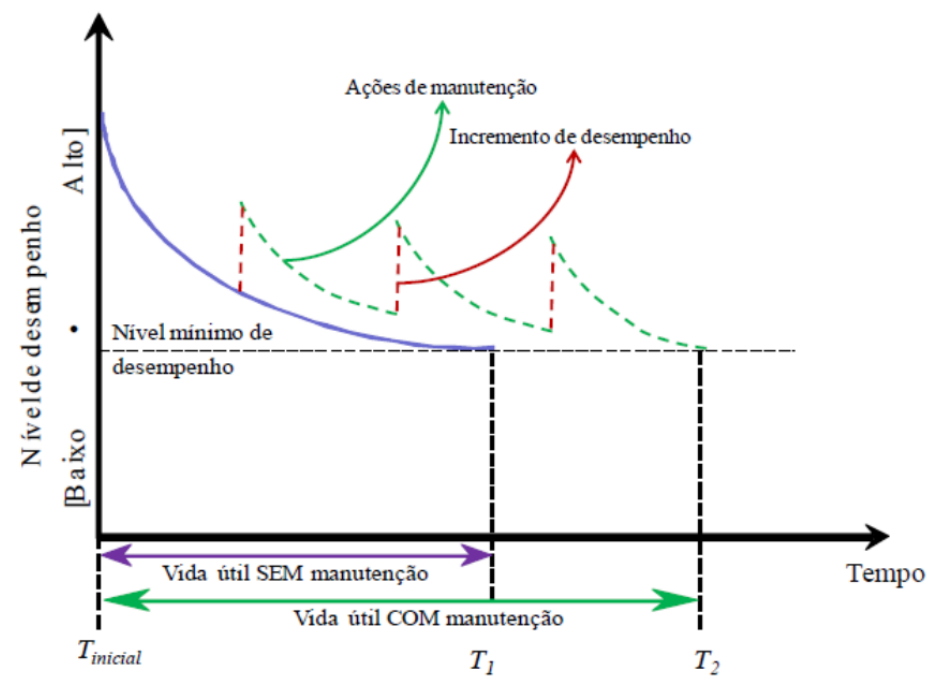

Figura 1 - Relação da vida útil da estrutura com e sem manutenção (POSSAN; DEMOLINER, 2013)

Segundo Moscoso (2017), um sistema de gerenciamento de OAEs possui um fluxo de atividades baseado em três componentes fundamentais: o banco de dados, a análise de dados e o suporte à decisão, conforme ilustrado na Figura 2. 


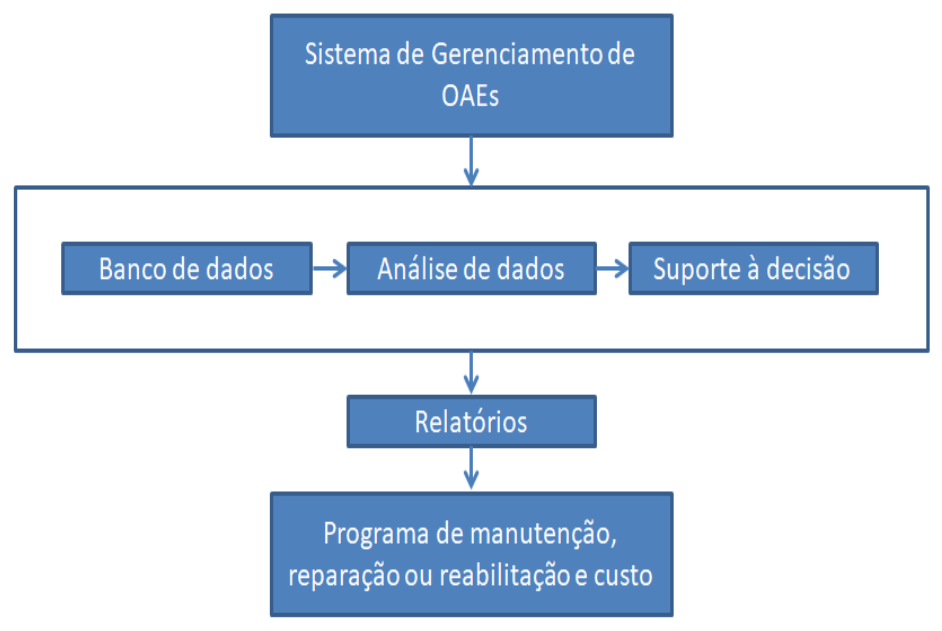

Figura 2 - Fluxo de atividades de um Sistema de Gerenciamento de OAEs (MOSCOSO, 2017)

Como mencionado anteriormente, as inspeções coletam os dados. Esses dados serão analisados e se transformarão em informação, servindo como base para a tomada de decisão concernente às ações necessárias na obra (MENDES, 2009). A partir disso, são produtos do sistema de gerenciamento os relatórios que irão instruir os programas de manutenção, reparação ou reabilitação.

Considerando que no Brasil, atualmente, são realizadas inspeções rotineiras a cada 2 anos nas OAEs em rodovias federais, o banco de dados está estabelecido no SGO, sistema administrado pelo DNIT. A partir disso, o próximo passo é a implementação de projetos de reabilitação para as obras avaliadas em condições críticas ou problemáticas.

Como as OAEs presentes na rede rodoviária brasileira passaram a receber inspeções e monitoramento constante recentemente, é provável que a demanda de obras que necessitam de recuperação seja elevada, fazendo com que seja necessário definir prioridades dentro do sistema de gerenciamento.

Nesse sentido, uma proposta para a gestão de reabilitação de OAEs é a atribuição de pesos que estabeleçam prioridades entre os diferentes critérios determinantes das condições críticas nas obras.

\section{Parâmetros de gestão para reabilitação}

Para determinação dos parâmetros de gestão foram atribuídos pesos com base em 4 critérios. Esses pesos variam de 1 a 32, e por meio da sua soma será possível visualizar a necessidade de recuperação da OAE.

Com as inspeções rotineiras são identificadas as obras em estado crítico, que possuem nota de estabilidade igual a 1. No caso dessas OAEs a intervenção deve ser imediata, sob risco iminente de acidentes. Dessa forma, o parâmetro de maior relevância para determinação da reabilitação é a nota de estabilidade.

Em seguida, uma característica importante a ser observada é a capacidade de carga para qual a estrutura foi projetada, sendo uma característica diretamente relacionada ao seu ano de construção. Quando uma OAE, construída antes de 1985, não passou por obras de reforço e alargamento, essa capacidade de carga está desatualizada para os padrões de tráfegos atuais, de 45 toneladas.

O próximo critério é a largura da seção, uma vez que as obras mais antigas não possuem a largura mínima estabelecida pela norma atual de $12,80 \mathrm{~m}$. Essas obras com essas características indicam riscos para os usuários, uma vez que há um estreitamento de pista e os dispositivos de segurança são guarda-corpos e guarda-rodas obsoletos, que não oferecem a mesma segurança que as barreiras New Jersey, indicadas nos projetos atuais.

Por último, também foi considerada a nota de conservação atribuída às OAEs. Essa nota indica a condição dos elementos não estruturais, mas que possuem danos que podem comprometer a segurança dos usuários, 
como guarda corpos rompidos, ou até a mesmo comprometer a estabilidade da OAE a longo prazo, como presença de processo de erosão nos aterros de acesso.

O Quadro 1 apresenta a distribuição dos pesos com base em cada critério. A partir desses valores, foi estabelecido que a obra cuja soma totalizar valores entre 39 e 56 deve ser considerada crítica, com necessidade de reabilitação imediata. Valores inferiores a 39 significam que a OAE deve estar em observação e receber processos de manutenção preventiva regularmente.

Quadro 1 - Parâmetros e pesos adotados

\begin{tabular}{|c|c|c|c|}
\hline Nota Estrutural & Peso & $\begin{array}{c}\text { Capacidade de } \\
\text { carga }\end{array}$ & Peso \\
\hline 1 & 32 & TB $24 \mathrm{tf}$ & 20 \\
\hline 2 & 30 & \multirow{2}{*}{ TB $36 \mathrm{tf}$} & \multirow{2}{*}{10} \\
\hline 3 & 12 & & \\
\hline 4 & 4 & \multirow{2}{*}{ TB 45tf } & \multirow{2}{*}{5} \\
\hline 5 & 1 & & \\
\hline $\begin{array}{l}\text { Largura da } \\
\text { seção }\end{array}$ & Peso & $\begin{array}{c}\text { Nota } \\
\text { Conservação }\end{array}$ & Peso \\
\hline Obras até $8,30 \mathrm{~m}$ & 4 & 1 & 5 \\
\hline Obras até $10,00 \mathrm{~m}$ & 3 & 2 & 4 \\
\hline Obras até $12,80 \mathrm{~m}$ & 2 & 3 & 3 \\
\hline \multirow{2}{*}{$\begin{array}{l}\text { Obras acima de } \\
12,80 \mathrm{~m}\end{array}$} & \multirow{2}{*}{1} & 4 & 2 \\
\hline & & 5 & 1 \\
\hline
\end{tabular}

\section{Estudos de Caso}

Para esse estudo, foram selecionadas 4 Obras de Arte Especiais, localizadas em estados diferentes e com características variadas. $O$ objetivo é aplicar os critérios estabelecidos na seção 5 a fim de exemplificar a aplicação dos parâmetros de gestão de reabilitação para essas obras. No Quadro 2 foram resumidas as principais informações das pontes.

Quadro 2 - Relação das OAEs avaliadas

\begin{tabular}{|c|c|c|c|c|c|c|c|}
\hline $\mathbf{n}$ o & Identificação & Estado & Rodovia & $\mathbf{k m}$ & $\begin{array}{c}\text { Extensão } \\
(\mathbf{m})\end{array}$ & Latitude & Longitude \\
\hline 1 & $\begin{array}{c}\text { Ponte sobre o Rio } \\
\text { Jequitinhonha }\end{array}$ & Bahia & BR-101 & 661,72 & 510,25 & $-15.949502^{\circ}$ & $-39.559105^{\circ}$ \\
\hline 2 & $\begin{array}{c}\text { Ponte sobre o Rio } \\
\text { dos Macacos }\end{array}$ & Goiás & BR-364 & 134,70 & 40,00 & $-18.199283^{\circ}$ & $-51.328717^{\circ}$ \\
\hline 3 & $\begin{array}{c}\text { Ponte sobre o Rio } \\
\text { Pindaré }\end{array}$ & Maranhão & BR-316 & 251,11 & 263,70 & $-3.659373^{\circ}$ & $-45.467302^{\circ}$ \\
\hline 4 & $\begin{array}{c}\text { Ponte sobre o Rio } \\
\text { Aruri }\end{array}$ & Pará & BR-163 & 514,17 & 167,40 & $-5.401769^{\circ}$ & $-55.883140^{\circ}$ \\
\hline
\end{tabular}


O Quadro 3 apresenta as informações das OAEs que são pertinentes à avaliação segundo os parâmetros estabelecidos na seção 5. Já no Quadro 4 foram atribuídos os pesos a cada parâmetro, possibilitando realizar o somatório destes e verificar as obras que devem ser reabilitadas imediatamente.

Quadro 3 - Informações para avaliação

\begin{tabular}{|c|c|c|c|c|}
\hline Ponte & $\begin{array}{c}\text { Nota } \\
\text { Estrutural }\end{array}$ & $\begin{array}{c}\text { Nota de } \\
\text { Conservação }\end{array}$ & $\begin{array}{c}\text { Capacidade de } \\
\text { Carga }\end{array}$ & Largura (m) \\
\hline 1 & 1 & 1 & TB 36 & 9,80 \\
\hline 2 & 3 & 3 & TB 24 & 8,20 \\
\hline 3 & 3 & 2 & TB 36 & 9,54 \\
\hline 4 & 2 & 1 & TB 45 & 13,00 \\
\hline
\end{tabular}

Quadro 4 - Pesos atribuídos

\begin{tabular}{|c|c|c|c|c|c|}
\hline Ponte & $\begin{array}{c}\text { Nota } \\
\text { Estrutural }\end{array}$ & $\begin{array}{c}\text { Nota de } \\
\text { Conservação }\end{array}$ & $\begin{array}{c}\text { Capacidade de } \\
\text { Carga }\end{array}$ & Largura (m) & Somatório \\
\hline 1 & 32 & 5 & 10 & 3 & 50 \\
\hline 2 & 12 & 3 & 20 & 4 & 39 \\
\hline 3 & 12 & 4 & 10 & 3 & 29 \\
\hline 4 & 30 & 5 & 5 & 1 & 41 \\
\hline
\end{tabular}

Analisando os valores obtidos, constata-se que as pontes sobre os rios Jequitinhonha, Aruri e Macacos, números 1, 2 e 4 no Quadro 2, encontram-se dentro da escala crítica adotada nesse artigo. Percebe-se que a Ponte sobre o Rio Aruri, apesar de ter sido construída mais recentemente e possuir largura e trem-tipo atualizados, apresenta uma situação problemática nos elementos estruturais e de conservação, justificando a necessidade de reabilitação. Já as Pontes sobre o Rios Jequitinhonha e Macacos necessitam de intervenções, atendo as questões funcionais de alargamento e aumento da capacidade de carga. Cabe destacar que a Ponte sobre o Rio Jequitinhonha além de possuir nota crítica estrutural, sua nota de conservação é 1 , o que indica que o tempo em exercício está refletindo na estabilidade da estrutura.

Já a Ponte sobre o Rio Pindaré, necessita de observação constante de acordo com o somatório de pesos encontrado. Observa-se que a obra foi construída antes de 1985, onde apresenta deficiências funcionais e insuficiências estruturais, como largura e trem tipo inferior em desacordo com os normativos vigentes nacionais.

\section{Conclusão}

Para uma gestão de reabilitação efetiva em OAEs é necessário um banco de dados completo e atualizado, pois, é a partir das informações das inspeções que será possível comparar os critérios determinantes para a necessidade ou não de recuperação e reabilitação da estrutura. Dessa forma, as inspeções rotineiras devem ser realizadas na periodicidade recomendada, de forma completa, com o registro do máximo de informações possíveis.

Outro fator importante, é que as inspeções necessitam ser menos subjetivas, evitando que o entendimento das notas atribuídas varie entre os engenheiros. Uma alternativa para tal é a inserção da temática de inspeção de pontes e viadutos no currículo de formação dos engenheiros civis, e uma maior disseminação de cursos e disciplinas relacionadas ao tema.

Vale destacar que para a implantação de um sistema de gerenciamento de Obras de Arte Especiais, necessitase de conhecimento de várias disciplinas, como estruturas, drenagem, sinalização, dispositivos de segurança, 
entre outras, sendo necessário uma análise a fundo na identificação de todas as deficiências funcionais interligadas as insuficiências estruturais.

No entanto, faz-se cada vez mais necessário o uso de recursos tecnológicos para aprimorar as práticas da engenharia, sendo softwares e aplicativos ferramentas indispensáveis atualmente para diversas atividades. No caso do gerenciamento de OAEs, percebe-se que ainda há muito que evoluir para que os sistemas possam ser mais eficazes, de forma que facilite a visualização e integração de informações pertinentes, tanto para a realização da inspeção, quanto para análise e interpretação posterior.

Com isso, é possível obter os dados necessários para realizar a gestão de reabilitação das OAEs no Brasil, prevenindo possíveis colapsos das estruturas em situação crítica. Além disso, a partir da atribuição de parâmetros proposta nesse estudo, é possível realizar uma análise mais precisa das informações obtidas ao longo do tempo, adotando critérios que interferem diretamente nas condições das pontes e avaliando de forma menos subjetiva a necessidade de reabilitação das estruturas.

Uma proposta é a integração de parâmetros de gestão como o apresentado aos sistemas existentes, permitindo uma sinalização automática da condição da $\mathrm{OAE}$, tornando assim o processo mais ágil e eficaz.

\section{Referências Bibliográficas}

ABNT. (2019). NBR 9452: Inspeção de pontes, viadutos e passarelas de concreto. Rio de Janeiro, 2019.

ASHWELL, E. Highways England gives bridge inspections a major digital overhaul. [S. I.], 22 out. 2019. Disponível em: https://www.newcivilengineer.com/latest/highways-england-gives-bridge-inspectionsmajor-digital-overhaul-22-10-2019/. Acesso em: 22 dez. 2020.

BENTLEY. AssetWise for Roads and Bridges. [S. I.: s. n.], 2020. Disponível em: https://www.bentley.com/en/products/product-line/asset-performance/assetwise-asset-reliability. Acesso em: 22 dez. 2020.

CARVALHO, D. M. (2018). Análise de desempenho das intervenções de alargamento e reforço de pontes rodoviárias de concreto armado em obras de duplicação de rodovias. Tese de mestrado. Escola de Engenharia, Universidade Federal de Minas Gerais, Belo Horizonte, 2018. 253p.

CEREMA (2018). Surveillance et entretien courant des ouvrages d'art routiers. França, set. 2018.

DNIT. (2004). Manual de inspeção de pontes rodoviárias. Rio de Janeiro, 2004.

LIMONGELLI, M. P.; ORCESI, A. Report of the innovation subgroup: COST ACTION TU 1406. [S. I.: s. n.], março 2019.

MENDES, P. de T. C. (2009). Contribuições para um modelo de gestão de pontes de concreto aplicado à rede de rodovias brasileiras. 234 f. Tese (Doutorado). Escola Politécnica, Universidade de São Paulo, São Paulo, 2009.

MOSCOSO, Y. F. M. (2017). Modelos de Degradação para Aplicação em Sistemas de Obras de Arte Especiais - OAEs. Tese de Doutorado em Estruturas e Construção Civil, Publicação E.TD-3A/17, Departamento de Engenharia Civil e Ambiental, Universidade de Brasília, Brasília, DF, 185p.

POSSAN, E.; DEMOLINER, C. A. (2013). Desempenho, Durabilidade e Vida Útil das Edificações: Abordagem Geral. Revista Técnico-Científica do CREA-PR, 2013.

VITÓRIO, J. A. P. (2006). Vistorias, Conservação e Gestão de Pontes e Viadutos de Concreto. 48o Congresso Brasileiro do Concreto. Rio de Janeiro, IBRACON, 2006.

VITÓRIO, J. A. P. (2007). Acidentes Estruturais em Pontes Rodoviárias - Causas, diagnósticos e soluções. II Congresso Brasileiro de Pontes e Estruturas. Rio de Janeiro, 2007.

VITÓRIO, J. A. P. (2013). Um estudo comparativo sobre métodos de alargamento de pontes rodoviárias de concreto armado, com a utilização das normas brasileiras e eurocódigos. Tese de doutorado. Universidade do Porto, Portugal, 2013. 296p. 